\title{
Lidil
}

Revue de linguistique et de didactique des langues

$41 \mid 2010$

Énonciation et rhétorique dans l'écrit scientifique

\section{Pour une approche énonciative de l'écrit scientifique}

\section{Françoise Boch et Fanny Rinck}

\section{(2) OpenEdition}

1 Journals

Édition électronique

URL : http://journals.openedition.org/lidil/3004

DOI : 10.4000/lidil.3004

ISSN : 1960-6052

Éditeur

UGA Éditions/Université Grenoble Alpes

\section{Édition imprimée}

Date de publication : 30 mai 2010

Pagination : 5-14

ISBN : 978-2-84310-167-0

ISSN : $1146-6480$

\section{Référence électronique}

Françoise Boch et Fanny Rinck, « Pour une approche énonciative de l'écrit scientifique », Lidil [En ligne], 41 | 2010, mis en ligne le 30 novembre 2011, consulté le 21 septembre 2020. URL : http:// journals.openedition.org/lidil/3004; DOI : https://doi.org/10.4000/lidil.3004 


\title{
Pour une approche énonciative de l'écrit scientifique
}

\author{
Françoise Boch* et Fanny Rinck**
}

Ce numéro réunit sept contributions analysant l'écrit scientifique dans une perspective linguistique. Il s'intéresse aux approches énonciatives et rhétoriques des textes en lien avec l'activité scientifique et ses enjeux sociaux.

On entendra ici le discours scientifique comme discours produit dans le cadre de l'activité de recherche à des fins de construction et de diffusion du savoir. Si les sciences dures ont été jusqu'à présent plus explorées que les sciences appliquées, humaines ou sociales, les différences disciplinaires sont aujourd'hui considérées comme essentielles, de même que la diversité des genres, qui renvoie à la diversité des activités dans le monde de la recherche et notamment à la question de savoir comment elles se situent par rapport à la logique de la découverte et la logique de l'exposition.

$\mathrm{Au}$ plan méthodologique, on notera les liens de plus en plus serrés entre le champ de recherche du discours scientifique et la linguistique de corpus, les analyses linguistiques exploitant de plus en plus les avancées techniques et théoriques que connait actuellement la linguistique de corpus, champ en pleine expansion. Citons à ce propos la constitution récente ${ }^{1} \mathrm{~d}^{\prime}$ un vaste corpus d'écrits scientifiques (thèses, articles, mémoires d'habilitation à diriger des recherches) dans plusieurs disciplines (linguistique, sciences de l'éducation, biologie, médecine, etc.) en français et en anglais, disponible en accès libre sur le site Scientext ${ }^{2}$,

* LIDILEM, EA 609, Université Stendhal-Grenoble 3.

** MoDyCo, UMR 7114, Université Paris 10.

1. Projet Scientext (responsables : F. Grossmann et A. Tutin, LIDILEM), financé par l'ANR «Corpus», 2007-2010.

2. «Un corpus et des outils pour étudier le positionnement et le raisonnement dans les écrits scientifiques » [en ligne], disponible sur $<$ http://scientext.mshalpes.fr/scientext-site/spip.php?article1> [consulté en avril 2010]. 
où l'on trouvera également des outils permettant des requêtes variées sur les textes. Cela étant, les approches sur corpus ne portent pas toutes sur des ensembles de textes mais peuvent relever d'études plus qualitatives, résultant pour certaines d'une démarche ethnographique menée in situ, au sein par exemple de réunions de travail entre chercheurs ${ }^{3}$.

L'intérêt pour le discours scientifique n'est pas nouveau mais connait aujourd'hui un essor considérable; si ces travaux relèvent de cadres variés (cf. pour un état des lieux, Rinck, 2010), on peut toutefois distinguer à grands traits deux types d'études, celles s'inscrivant en didactique, les autres en analyse de discours.

Les premières visent à former à la communication en langue étrangère, dans les champs du Français sur Objectifs spécifiques, et l'English for Academic Purposes ainsi qu'à l'écrit académique, à travers les courants anglo-saxons Writing in the Disciplines, Writing Accross the Curriculum, College Composition Studies, et dans la francophonie la Didactique de l'écrit dans l'enseignement supérieur ${ }^{4}$, ou encore Littéracies Universitaires, selon une appellation récente, qui tend à se généraliser aujourd'hui ${ }^{5}$.

Les secondes visent à cerner, à travers la description linguistique, les fondements des communautés discursives; c'est dans cette perspective que s'inscrit le présent numéro. Deux champs linguistiques sont mobilisés, chacun dans une acception large, la rhétorique et la linguistique de l'énonciation.

L'approche rhétorique du discours scientifique est fondée dans les années 1960 sur une revendication récurrente, autour de laquelle ont convergé des approches historiques, sociologiques et ethnographiques, avec pour finalité de promouvoir une conception constructiviste de la science. De nombreux travaux (voir par exemple Latour et Fabbri,

3. Cf. le numéro à paraitre «Les discours scientifiques - Des marques linguistiques aux épistémologies» de la Revue d'Anthropologie des connaissances, coordonné par F. Grossmann.

4. Voir notamment Boch et Grossmann (2001); Delcambre et Jovenet (2002); Pollet et Boch (2002); Boch, Laborde et Reuter (2004); Kara (2004); Reuter (2004).

5. Cf. à ce sujet le projet Écrits Universitaires : Inventaires, Pratiques, Modèles (responsables : I. Delcambre, Théodile, et F. Boch, LIDILEM, financé par l'ANR «Apprentissages» 2007-2010), qui se clôt par l'organisation du colloque international «Littéracies universitaires : Savoirs, Écrits, Disciplines », septembre 2010, Lille. 
1977; Prelli, 1989; Gross, 1990 et pour des travaux plus récents Hyland, 2002; Harwood, 2005; Grossmann et Rinck, 2004 ; Fløttum, 2007) ont étayé l'idée désormais bien établie que, loin d'être neutres et objectifs, les textes scientifiques comportent une forme de subjectivité et une visée persuasive.

Il reste toutefois à étayer le type de subjectivité auquel on a affaire, la construction discursive du savoir scientifique et la dimension argumentative (Amossy, 2000) de textes qu'il faut concevoir à la fois comme des manières de dire et de faire la recherche (cf. notamment Beacco, 1992; Carter, 2007). La rhétorique, mais aussi la sémiotique, l'analyse de discours et la pragmatique peuvent y contribuer, et éclairer les enjeux cognitifs, sociaux et praxéologiques des textes en lien avec leurs caractéristiques, et en particulier leurs caractéristiques énonciatives.

Parallèlement en effet, le champ de l'énonciation dans l'écrit scientifique et plus largement dans les textes argumentatifs fait l'objet d'un grand intérêt en linguistique, notamment autour de la polyphonie. On peut citer, entre autres, les activités du groupe Ci-dit sur le discours rapporté et la circulation des discours, les approches développées dans la théorie scandinave de la polyphonie par H. Nølke, K. Fløttum, C. Noren (2000), l'analyse énonciative du point de vue chez A. Rabatel (1998, 2003, 2004), les travaux de R. Amossy et de R. Koren sur l'ethos dans son lien avec l'argumentation et la responsabilité (Amossy, 1999; Amossy et Koren, 2004).

Dans les contributions que réunit ce numéro, plusieurs axes d'analyse sont traités, qui relèvent du type de discours étudié et du positionnement de l'auteur dans son écrit.

\section{Types de discours étudiés}

L'écrit scientifique est notamment étudié à travers le genre emblématique de l'article, mais aussi à travers les comptes rendus d'ouvrages (F. Salager, M. Á. Alcaraz-Ariza et M. Pabón). L'accent est mis sur la diversité des normes en usage, en diachronie en ce qui concerne les comptes rendus d'ouvrage étudiés de la fin du XIX siècle au début du $\mathrm{XXI}^{\mathrm{e}}$, et, en ce qui concerne le genre de l'article, en fonction de styles individuels (U. Reutner), en fonction des disciplines (A. Tutin) et en fonction du statut de l'auteur (K. Fløttum et E. T. Vold).

En analyse de discours, le discours scientifique est considéré comme un discours «fermé» (Charaudeau et Maingueneau, 2002, p. 261) car, 
dans un domaine donné, ceux qui en sont à l'origine sont peu ou prou ceux auxquels il est adressé. Cependant, la «communauté discursive» (Swales, 1990) ainsi constituée demande à être envisagée de manière dynamique. Notre numéro se clôt sur cette question (T. Donahue) en traitant de l'acculturation des étudiants aux discours universitaires, expression qui traduit mal le courant de l'«academic discourse». Peu utilisée en contexte francophone en raison vraisemblablement des ambigüités attachées au terme «académique », cette désignation l'emporte en contexte anglophone où «scientific discourse» ne concerne que les disciplines de sciences dures. L' «academic discourse» prend en compte conjointement le discours des chercheurs, sa transposition didactique à l'université et les écrits des étudiants dans leur parcours universitaire et leur formation à la recherche.

\section{Le positionnement dans l'écrit scientifique}

Les contributions ont en commun un intérêt pour la question du positionnement dans l'écrit scientifique. La notion de positionnement est envisagée sous deux angles, dans sa composante socio-institutionnelle d'une part et linguistique d'autre part. C'est d'abord à travers la comparaison entre articles de doctorants et articles de leurs ainés (K. Fløttum et E. T. Vold) qu'est étudiée cette composante socio-institutionnelle, puis à travers une recherche sur l'acculturation à l'écrit académique (T. Donahue). Dans les deux études, l'enjeu est en effet de comprendre les pratiques scripturales en termes d'acculturation, en fonction du statut du scripteur dans le monde académique, d'où l'intérêt pour les étudiants qui en découvrent peu à peu les normes et pour les doctorants, à la frontière institutionnelle qui fait passer du statut d'étudiant à celui de chercheur ou d'enseignant-chercheur.

Au plan linguistique, les contributions éclairent différents procédés aux fondements du positionnement du scripteur dans l'écrit scientifique. À l'encontre de la vision de l'écrit scientifique comme écrit neutre et objectif, mais aussi du simple contre-pied de cette thèse, elles montrent en quoi le positionnement est le résultat de procédés d'effacement énonciatif et de procédés de marquage de l'attitude ou de la subjectivité du locuteur.

Le rôle des pronoms personnels dans le discours scientifique est traité dans plusieurs articles. Il s'agit d'aller au-delà du constat de la présence de traces de l'auteur dans l'usage de ces pronoms pour les étudier en termes de choix stratégiques à l'aide de questionnaires 
(U. Reutner) et comme des composantes des images et rôles d'auteur qui se construisent dans le texte (A. Tutin; K. Fløttum et E. T. Vold; F. Salager, M. Á. Alcaraz-Ariza et M. Pabón), de manière diverse en fonction des époques (F. Salager, M. Á. Alcaraz-Ariza et M. Pabón), des disciplines (A. Tutin) et du statut de l'auteur (K. Fløttum et E. T. Vold). Ces articles montrent la diversité des valeurs des pronoms et les difficultés de l'interprétation (notamment dans le cas du «nous» académique renvoyant au seul auteur et de «nous» incluant le lecteur/la communauté de chercheurs). Ils envisagent les pronoms dans leur contexte, en association avec d'autres observations linguistiques, comme les indications données sur l'auteur du compte rendu d'ouvrage et de l'ouvrage recensé (F. Salager, M. Á. Alcaraz-Ariza et M. Pabón), par exemple leur rattachement institutionnel, ou le lexique assurant le guidage du lecteur, l'argumentation en faveur des positions de l'auteur, l'explicitation des procédures de la recherche (K. Fløttum et E. T. Vold).

$\mathrm{Si}$ les pronoms personnels représentent une entrée traditionnellement prise en compte en linguistique énonciative et dans les écrits scientifiques, c'est plus largement le lexique et la phraséologie qui sont explorés dans les études réunies ici. L'interface entre la syntaxe et la sémantique et les effets pragmatiques des patrons phraséologiques sont au cœur de deux contributions (Z. Livnat; I. Kanté). La première (Z. Livnat) étudie la rhétorique de l'objectivité à travers les constructions caractéristiques de l'effacement énonciatif du discours scientifique, comme le passif et les nominalisations. La seconde (I. Kanté) se centre sur les noms-têtes de subordonnées complétives comme marqueurs modaux qui peuvent prendre une valeur épistémique ou évaluative dans le discours scientifique en anglais.

\section{Présentation du dossier}

Agnès Tutin explore la question de la présence et du positionnement de l'auteur dans l'article à travers l'étude des verbes de positionnement associés à un pronom sujet (ex. : je cherche à démontrer). Elle mène une comparaison fine de parties textuelles (introductions et conclusions) extraites de 60 articles de trois disciplines (linguistique, psychologie et sciences de l'éducation). Les résultats de sa première étude sur les pronoms montrent que les linguistes sont ceux qui expriment le plus leurs voix personnelles. Sa deuxième étude portant sur les verbes de positionnement permet de dégager deux tendances : si les psychologues mettent en avant les hypothèses et les résultats, les chercheurs en 
sciences de l'éducation insistent davantage sur les intentions, opinions et questionnements du chercheur, tandis que les linguistes se situent entre ces deux tendances marquées en faisant usage de ces deux grands types de verbes de positionnement. À l'issue de cette double étude, A. Tutin constate une grande hétérogénéité des usages, à la fois au sein de la discipline et entre les disciplines, ce qui rend l'étiquette «sciences humaines» peu pertinente.

K. Fløttum et E. T. Vold s'intéressent au genre de l'article de recherche en français dans le domaine de la linguistique et proposent une comparaison entre des articles publiés par des doctorants et ceux de chercheurs plus expérimentés. Leur analyse se centre sur la construction de l'ethos auto-attribué, autrement dit l'image du scripteur dans son texte, telle qu'elle se manifeste à travers l'usage des pronoms personnels. Les pronoms je, nous et on, permettent aussi de pointer, en fonction des verbes avec lesquels ils se combinent, les rôles (de chercheur, de scripteur ou d'argumentateur) endossés par l'auteur dans son texte. Une première approche quantitative de l'usage des pronoms et des rôles d'auteur dans les articles de doctorants et ceux de leurs aînés est complétée par l'étude qualitative de deux articles de doctorants, qui met en évidence leur souci de guider le lecteur et de rendre explicites les procédures de leur recherche.

F. Salager, M. Á. Alcaraz Ariza et M. Pabón proposent une étude en diachronie du genre de la recension d'ouvrages. Leur corpus se constitue de recensions publiées dans des revues de médecine à trois périodes différentes : la fin du $\mathrm{XIX}^{\mathrm{e}}$ siècle, le milieu du $\mathrm{Xx}^{\mathrm{e}}$ et le début du $\mathrm{XXI}^{\mathrm{e}}$. L'analyse porte sur les formes d'identification de l'auteur de l'ouvrage recensé et les manifestations de l'auteur de la recension. Cette étude met en évidence un double changement dans l'écriture des recensions : les informations servant à identifier les auteurs des ouvrages recensés se font de plus en plus succinctes et l'accent est davantage mis sur l'ouvrage que sur son auteur; inversement, les informations servant à identifier l'auteur de la recension se développent et l'usage de «we» cède la place à l'usage de «I». Le genre est ainsi questionné en regard de l'évolution de la communauté scientifique et de l'influence des langues qui y sont en usage.

U. Reutner revisite la question de la présence des pronoms personnels dans l'article, afin de mesurer l'évolution de ces usages par rapport à ce qu'elle appelle «l'ancien tabou du moi», qui exige traditionnellement que soit effacé du discours scientifique les marques personnelles de l'auteur, au nom d'un idéal d'objectivité de la recherche et de la 
modestie du chercheur. Dans cette perspective, son analyse s'appuie à la fois sur un examen de pratiques (articles dans le champ des études littéraires et linguistiques) et sur les résultats d'une enquête menée auprès d'un grand échantillon de chercheurs en linguistique, visant à identifier leurs choix conscients de pronoms utilisés, et en particulier leurs éventuelles stratégies d'évitement du je. Ses observations convergent : si la présence des pronoms personnels (je, on, nous) dans les textes est attestée, elle est extrêmement variable selon les auteurs. Ces différences inter- (voire intra) textuelles sont interprétées par U. Reutner comme le résultat de préférences individuelles. Les réponses obtenues par les chercheurs tendent également à renforcer l'idée que ces usages sont fortement dépendants du style individuel de l'auteur. Elle en conclut qu'en l'absence «d'idéal discursif» commun, il est peu envisageable de déduire des usages experts des recommandations rédactionnelles destinées aux apprentis-chercheurs.

Z. Livnat s'intéresse à la rhétorique de l'objectivité à partir d'un corpus d'articles en hébreu dans le domaine des sciences sociales. Il inventorie les constructions dites «impersonnelles» au sens où elles se caractérisent par un effacement du locuteur : l'usage d'opérateurs modaux ou évaluatifs suivis d'un infinitif, possible dans certaines langues comme l'hébreu, la voix passive et les métaphores grammaticales. Ces dernières, définies en référence à M. K. Halliday, sont analysées à travers deux exemples, la nominalisation et les métonymies, qui permettent de donner un sujet inanimé à un verbe désignant une activité cognitive (par exemple «ce papier discute»). L'auteur traite ainsi de la construction des faits scientifiques et de la manière dont l'argumentation s'inscrit dans la langue, à partir d'une approche à la charnière de la syntaxe et de la sémantique.

I. Kanté n'analyse pas le discours scientifique en lui-même, mais en le comparant au discours juridique, avec l'hypothèse que l'usage des formes linguistiques varie en fonction des logiques propres aux différents domaines d'activité. Il se centre sur les noms-têtes de phrases en que $\mathrm{P}$ (ou «that clauses»), de type «fact», «evidence», «statement», qui se définissent comme des formes d'expression nominale de la modalité : ils signalent le positionnement du scripteur et caractérisent le contenu propositionnel. Prenant comme point de départ un inventaire des différents patrons phraséologiques qui mobilisent les noms-têtes, l'étude compare leurs occurrences dans les deux corpus scientifique et juridique. Elle met en évidence que les noms-têtes sont particulièrement fréquents dans le discours scientifique et analyse leur valeur modale 
dans les deux corpus en distinguant les épistémiques, les déontiques et les évaluatifs. Non seulement les deux corpus ne recourent pas aux mêmes patrons, mais les mêmes patrons servent des fonctions pragmatiques différentes.

T. Donahue s'interroge sur l'évolution du rapport aux écrits disciplinaires que connaissent les étudiants tout au long de leur cursus, ici dans une université américaine. À cette fin, elle compare deux types de données : elle mène d'une part une analyse des pratiques scripturales réalisées par trois étudiants durant leurs quatre années d'étude; d'autre part, elle effectue une analyse de contenus d'une série d'entretiens (réalisés à deux moments-clés du cursus) auprès de ces mêmes étudiants. À partir de cette étude contrastive, elle met en lumière un décalage important entre l'évolution de la qualité des écrits estudiantins, marquée par une appropriation progressive des contenus disciplinaires et des conventions à l'œuvre dans la discipline concernant les écrits qui la caractérisent, et la difficulté qu'ont manifestement ces mêmes étudiants à verbaliser cette évolution, dont ils n'ont qu'une conscience assez limitée.

En somme, ce numéro permet d'étayer la question des genres de discours et celle du positionnement dans ses fondements linguistiques, en mettant en évidence comment il se construit, à la fois à travers les traces de l'auteur et les formes d'effacement. On y discute de l'influence des genres et des langues sur les pratiques scripturales et la part de conscience quant aux choix effectués. Ce numéro montre en outre en quoi le positionnement affecte aussi bien la dimension persuasive de l'écrit scientifique que sa dimension épistémique : le positionnement et les rôles de l'auteur dans son texte, en opposition à une vision «ornementale» de la rhétorique scientifique, se caractérisent par des logiques propres aux disciplines dans leurs modes de construction des connaissances et par les habitus, linguistiquement marqués, qui permettent d'asseoir la validité de ces dernières.

\section{RÉFÉRENCES BIBLIOGRAPHIQUES}

Amossy R. (éd.) (1999) : Images de soi dans le discours. La construction de l'ethos, Genève, Delachaux et Niestlé.

Amossy R. (2000) : L'argumentation dans le discours. Discours politique, littérature, d'idées, fiction, Paris, Nathan. 
Amossy R. et Koren R. (2004) : Argumentation et prise de position. Pratiques discursives, Semen 17, Revue de Sémio-linguistique des textes et discours, Annales littéraires de l'Université de Franche-Comté.

BeAcCo J. C. (éd.) (1992) : «Les genres textuels dans l'analyse du discours : écriture légitime et communautés translangagières », Langages, $\mathrm{n}^{\circ} 105$, «Ethnolinguistique de l'écrit», p. 8-27.

Boch F. et Grossamnn F. (éds) (2001) : «Apprendre à citer le discours d'autrui», Lidil, ${ }^{\circ}$ 24, Grenoble, Université Stendhal.

Boch F., Laborde-Milaa et Reuter Y. (éds) (2004) : «Les écrits universitaires », Pratiques, n 121/122, Metz, Cresef.

Boch F. et Grossamnn F. (éds) (2001) : «Apprendre à citer le discours d'autrui», Lidil, $\mathrm{n}^{\circ}$ 24, Grenoble, Université Stendhal.

CARTER M. (2007) : «Ways of knowing, doing and writing in the disciplines», College Composition and Communication, vol. 58, n ${ }^{\circ} 3$, p. 385-418.

Charaudeau P. et Maingueneau D. (2002) : Dictionnaire d'analyse du discours, Paris, Seuil.

Ci-Diт, Groupe de recherche international et interdisciplinaire sur la circulation des discours, <http://www.ulb.ac.be/philo/serlifra/ci-dit/>.

Delcambre I. et Jovenet A. M. (éd.) (2002) : «Lire-écrire dans le supérieur», Spirale, $\mathrm{n}^{\circ} 29$, Lille 3.

FLøттм K. (2007) : Language and Discipline Perspectives on Academic Discourse, Cambridge, Cambridge Scholars Publishing.

Grossmann F. et Rinck F. (2004) : «La surénonciation comme norme du genre. L'exemple de l'article de recherche et du dictionnaire en linguistique », Langages, $\mathrm{n}^{\circ} 156$, p. 34-50.

Gross A. G. (1990) : The Rhetoric of Science, Cambridge, Harvard University Press.

HaRwOOD N. (2005) : «We do not seem to have a theory ... The theory I present here attempts to fill this gap: inclusive and exclusive pronouns in academic writing », Applied Linguistics, vol. 26, $\mathrm{n}^{\circ}$ 3, p. 343-375.

Hyland K. (2002) : "Authority and invisibility: authorial identity in academic writing », Journal of pragmatics, vol. 34, p. 1091-1112.

KARA M. (2004) : «Pratiques de la citation dans les mémoires de maitrise», Pratiques, ${ }^{\circ}$ 121/122, Metz, Cresef, p. 111-142.

LATOuR B. et FABBRI P. (1977) : «La rhétorique de la science : pouvoir et devoir dans un article scientifique», Actes de la recherche en sciences sociales, vol. 13, p. 81-95.

Nølke H., Fløttum K. et Noren C. (2004) : ScaPoLine. La théorie scandinave de la polyphonie linguistique, Paris, Kimé. 
Pollet M. C. et Boch F. (éds) (2002) : «L'écrit dans l'enseignement supérieur », Enjeux, Namur, CEDOCEF, n ${ }^{\circ}$ 53-54.

Prelli L. J. (1989) : A Rhetoric of Science: Inventing Scientific Discourse, University of South Carolina Press.

Rabatel A. (1998) : La construction textuelle du point de vue, Lausanne, Paris, Delachaux et Niestlé.

Rabatel A. (éd.) (2003) : «Le point de vue», Cahiers de Praxématique, $n^{\circ} 41$, Université Montpellier 3.

Rabatel A. (éd.) (2004) : «L'effacement énonciatif dans les discours représentés : effets pragmatiques de sur- et de sous-énonciation », Langages, $n^{\circ} 156$, Paris, Larousse.

Reuter Y. (2004) : «Analyser les problèmes de l'écriture de recherche en formation», Pratiques, ${ }^{\circ} 121 / 122$, p. 9-27.

RINCK F. (2006): L'article de recherche en Sciences du langage et en Lettres. Figure de l'auteur et identité disciplinaire du genre, Thèse de doctorat de l'Université Grenoble 3.

RincK F. (2010, sous presse): "L'analyse linguistique des enjeux de connaissance dans le discours scientifique. Un état des lieux», Revue d'Anthropologie des connaissances.

Swales J. (1990) : Genre Analysis. English in Academic research Settings, Cambridge, Cambridge University press. 